\title{
Mast cells correlate with angiogenesis and poor outcome in stage I lung adenocarcinoma
}

\author{
A. Imada, N. Shijubo, H. Kojima, S. Abe
}

Mast cells correlate with angiogenesis and poor outcome in stage I lung adenocarcinoma. A. Imada, N. Shijubo, H. Kojima, S. Abe. (C)ERS Journals Ltd 2000.

ABSTRACT: Angiogenesis is in part related to mast cells. However, the biological significance of mast cells within lung carcinoma remains unclear.

Immunohistochemistry was used to stain for tryptase, CD34 and vascular endothelial growth factor (VEGF) in 85 cases of stage I nonsmall cell lung carcinoma.

VEGF was found in 33 of 53 adenocarcinomas and 14 of 32 squamous cell carcinomas. Cases of adenocarcinoma had significantly higher mast cell counts than those of squamous cell carcinoma. In adenocarcinoma, mast cell counts in VEGFpositive tumours were significantly higher than in VEGF-negative tumours, whereas in squamous cell carcinoma they were not. Good correlation was observed between intratumoural mast cell counts and microvessel counts. Double staining showed most intratumoural mast cells expressed VEGF. Importantly, only in lung adenocarcinoma, members in the high mast cell count group had significantly worse prognosis than those in the low mast cell count group.

It is concluded that tumour-released vascular endothelial growth factors may be related to mast cell accumulation, intratumoural mast cells may produce vascular endothelial growth factor, and stromal mast cells correlate with angiogenesis and poor outcome in stage $I$ lung adenocarcinoma.

Eur Respir J 2000; 15: 1087-1093.

Invasion and metastasis are cardinal features of malignant tumours and the complexity of these phenomena is well recognized. Tumour cell interactions with interstitial stroma or specific host cells may be of prime importance in determining invasive and metastatic behaviour. Tumour angiogenesis is certainly one of the most important factors involved in the development and progression of some solid human tumours [1, 2]. Recent studies [3-7] have demonstrated that angiogenesis assessed by microvessel counts (MVCs) is closely related to postoperative relapse, especially with distal metastasis, indicating that MVC within tumours is a prognostic factor in nonsmall cell lung carcinoma (NSCLC), especially stage I NSCLC.

Increased numbers of mast cells have been shown in malignant tumours including colorectal carcinoma [8], breast carcinoma $[9,10]$, basal cell carcinoma of the skin [11], soft tissue tumours [12] and melanoma [13]. Although functional roles of mast cells in tumour behaviour have remained speculative, several lines of evidence [1419] suggest that mast cells are responsible for mediating angiogenesis.

Vascular endothelial growth factor (VEGF) is a highly specific mitogen for vascular endothelial cells, known to be involved in physiological (embryogenesis) and pathophysiological (rheumatoid arthritis, tumour) angiogenesis [20]. Recent investigations [4, 6, 7] have demonstrated that VEGF is one of the significant cytokines in the angiogenesis of lung carcinoma, and its expression in lung carcinoma cells correlates with clinical outcome. However mast cells have recently been shown to be a rich source of
VEGF $[17,18]$. In addition, VEGF is reported to induce chemotactic migration of mast cells [21]. The aims of this study were to assess intratumoural MVCs and mast cell counts, to examine VEGF expression, and to analyse the clinicopathological role of mast cell counts in patients with stage I NSCLC.

Methods

\section{Patients}

The subjects consisted of consecutive patients with stage I NSCLC who were treated only by surgery at Sapporo Medical University Hospital, (Sapporo, Japan) between January 1981 and December 1992. Lymph nodes were extensively sampled at thoracotomy and they were pathologically confirmed to have no metastasis in mediastinal lymph nodes. In this study, patients with atopic diseases including asthma, atopic dermatitis and allergic rhinitis were excluded in order to eliminate the influence of atopy on mast cell counts. Patients with stage I NSCLC who died of unrelated diseases were also excluded. The subjects consisted of 85 consecutive patients (56 male and 29 female); 58 patients had T1 tumour and 27 patients had T2 tumour. These tumours included 53 cases of adenocarcinoma, 32 cases of squamous cell carcinoma and none of large cell carcinoma. Forty-eight patients had well-differentiated carcinoma (30 patients adenocarcinoma and 18 squamous cell carcinoma), 28 patients had moderately-differentiated 
carcinoma ( 15 adenocarcinoma and 13 squamous cell carcinoma) and nine patients had poorly-differentiated carcinoma (8 adenocarcinoma and 1 squamous cell carcinoma). Postoperative follow-up comprised 3 or 6 monthly office visits, with yearly restaging or as indicated clinically. All reasonable attempts were made to confirm tumour recurrence/metastasis cytologically or histologically, using radiologically-guided or echo-guided fine needle aspiration or bronchoscopic biopsy. Twenty-six patients had postoperative relapse and 59 patients were disease-free and alive with a follow-up of 65-210 months (median, 137 months). When the survival rates were calculated using the Kaplan-Meier method, there were no significant differences in survivals rates between: patients with stage I lung adenocarcinoma and squamous cell carcinoma; patients that were histologically differentiated into subgroups of adenocarcinoma; and between moderate- and well-differentiation subgroups of squamous cell carcinoma.

\section{Immunohistochemistry}

Immunohistochemical analyses were performed on at least two paraffin blocks of resected lung tissue per patient. Details of the technique have been previously described for human mast cell tryptase [22], CD34 and VEGF [6]. Tumour tissue specimens were fixed with $10 \%$ buffered formalin and embedded in paraffin. Five-micrometrethick sections were mounted on silanized slides (Dako Japan, Kyoto, Japan) and deparaffinized with xylene and ethanol. To retrieve the antigen, sections were pretreated in $0.1 \%$ trypsin (Dako, Glostrup, Denmark) and 0.1\% $\mathrm{CaCl}_{2}$ in $50 \mathrm{mmol}$ Tris buffer $\mathrm{pH} 7.4$ at $37^{\circ} \mathrm{C}$ for $120 \mathrm{~min}$ before immunostaining of mast cell tryptase and pretreated in $10 \mathrm{mmol}$ citrate buffer, $\mathrm{pH} 6.0$ with autoclave for $15 \mathrm{~min}$ at $120^{\circ} \mathrm{C}$ before the immunostaining of CD34 as described previously [6]. No pretreatment was performed before the immunostaining of VEGF. The sections were then washed three times with phosphate buffered saline (PBS) for $5 \mathrm{~min}$. The sections were soaked in absolute methanol containing $0.3 \%$ hydrogen peroxide for 30 min at room temperature to remove endogenous peroxidase activity. To suppress nonspecific binding, the sections were incubated with $1.5 \%$ nonimmune goat serum for $20 \mathrm{~min}$. The sections were then incubated with mouse monoclonal antibody QBEND10 to CD34 (5 $\mu \mathrm{g} \cdot \mathrm{mL}^{-1}$; Immunotech, Cedex, France), with monoclonal antibody AA1 to human mast cell tryptase $\left(1 \mu \mathrm{g} \cdot \mathrm{mL}^{-1}\right.$; Dako), or with rabbit polyclonal antibody to VEGF $\left(5 \mu \mathrm{g} \cdot \mathrm{mL}^{-1}\right.$; Santa Cruz Biotechnology, Santa Cruz, CA, USA) for 60 min at room temperature. After being washed with PBS, the slides were subsequently incubated with biotin-conjugated goat antimouse or goat antirabbit immunoglobulin (Ig)G antibody for $30 \mathrm{~min}$. Then, after being washed again with PBS, the sections were incubated with avidinbiotin-peroxidase complex (Vectastain Elite ABC kit; Vector Laboratories, Berlingame, CA, USA) for $30 \mathrm{~min}$, and washed once more with PBS. The sections were finally incubated with $0.03 \%$ hydrogen peroxide and $0.05 \%$ 3,3'-diaminobenzidine. The slides were then washed in running tap water, counterstained with haematoxylin, and mounted in Canadian balsam. Nonimmunized mouse or rabbit IgG was used as a negative control. No significant immunohistochemical reaction occurred in the control sections.
The specificity of polyclonal antibody against VEGF was tested; VEGF blocking peptide (Santa Cruz Biotechnology) completely absorbed VEGF immunoreactivities in VEGF immunostaining.

To evaluate immunohistochemical expression of VEGF the authors established a score corresponding to the sum of both: staining intensity ( $0=$ negative; $1=$ weak; $2=$ intermediate; $3=$ strong); and percentage of positive cells $(0=$ $0 \%$ positive cells; $1=<25 \%$ positive cells; $2=26-50 \%$ positive cells; $3=>50 \%$ positive cells), as described elsewhere $[6,7]$. The sum of the staining intensity plus the percentage of positive cells reached a maximum score of 6 . A score $>3$ represented a positive immunohistochemical survey.

To assess intratumoural MVCs and mast cell counts, immunohistochemical reactivities for CD34 and tryptase were evaluated, respectively. The authors assessed delineated CD34-positive cells as a microvessel and tryptasepositive cells as mast cells. Mast cells were counted at the sites where mast cells most intensively accumulated in a $\times 200$ microscopic field (i.e., $\times 20$ objective lens and $\times 10$ ocular lens; $0.723 \mathrm{~mm}^{2}$. field of view ${ }^{-1}$ ). The authors excluded perivascular, peribronchial and peribronchiolar regions within tumours for the evaluation of mast cell counts. In the areas that were considered to be most active for neovascularization, stained vessels were counted in a $\times 200$ microscopic field; the MVCs were designated as MVCs at the most neovascularization sites. MVCs were also evaluated at the sites where mast cells most intensively accumulated in serial sections. The averages of mast cells and microvessels counted in five fields were calculated as described elsewhere [6]. The mast cell counts and MVCs were assessed without knowledge of patient outcome (the presence or absence of relapse or any other pertinent variable) by two investigators. Between two observers, there were good correlations of MVCs $(\mathrm{r}=0.866$, $\mathrm{p}<0.0001)$ and mast cell counts $(\mathrm{r}=0.950, \mathrm{p}<0.0001)$.

To assess whether intratumoural mast cells express VEGF, sections were double labelled with anti-VEGF plus antitryptase antibody using combined ABC-peroxidase and $\mathrm{ABC}$-alkaline phosphatase methods (Vectastain-AP kit, Vector Laboratories). Diaminobenzidine was used as the substrate of horseradish peroxidase and 5-bromo-4choloro-indolyl phosphate (BCIT)/nitroblue tetrazolium (NBT) (Vector Laboratories) was applied to the alkaline phosphatase substrate. For this experiment, several controls were used: 1) anti-VEGF antibody first and antitryptase antibody later; 2) antitryptase antibody first and anti-VEGF antibody later, and 3) omission of the first antibody and/or secondary antibody. To assess how many mast cells ex-press VEGF, VEGF-positive cells were counted in 200 tryptase-positive cells.

\section{Statistical analysis}

Data are expressed as mean \pm SD. The Mann-Whitney Utest was used for analysis of two unpaired samples and the Pearson's least squares linear regression analysis was used to determine correlations. Contingency tables were analysed for trends with the Chi-squared test. Survivals were calculated from the day of operation using the KaplanMeier method and differences in the survivals were 
examined by the Wilcoxon test. The level of critical significance was assigned at $\mathrm{p}<0.05$.

\section{Results}

\section{Microvessel counts}

The MVCs were assessed in the most intensive areas of neovascularization within tumours by an immunohistochemical survey of CD34. Delineated CD34-positive cells were counted as a microvessel (fig. 1A and 1B). The mean MVC in cases of adenocarcinoma was $79.8 \pm 39.4$ per $\times 200$ microscopic field with a range of 4-229 and that of squamous cell carcinoma was $33.8 \pm 15.7$ with a range of 5-72. The mean MVC at the most neovascularized sites in stage I lung adenocarcinoma was significantly higher than that in stage I lung squamous cell carcinoma $(\mathrm{p}<$ 0.0001 ). There were no significant differences of MVCs between genders, distinct tumour size $(\geq 3 \mathrm{~cm}$ and $<3$ $\mathrm{cm}$ ), or distinct histological differentiation subgroups in lung adenocarcinoma or in squamous cell carcinoma.

\section{Mast cell counts}

The authors assessed mast cell counts in the control lung tissue and at the sites where mast cells most intensively accumulated within tumours by an immunohistochemical survey of tryptase. Tryptase-positive cells were counted as mast cells (fig. 1C and 1D). In the control lung tissue the average mast cell count was $9.2 \pm 2.3$ per $\times 200$ microscopic field. The mean mast cell count in cases of adenocarcinoma was $84.9 \pm 71.5$ with a range of $1-284$ and that of squamous cell carcinoma was $18.7 \pm 15.6$ with a range of $1-77$. The mast cell counts in cases of adenocarcinoma were significantly higher than those of squamous cell carcinoma $(\mathrm{p}<0.0001)$. Most intratumoural mast cells were located proximally to microvessels in lung adenocarcinoma, whereas a few intratumoural mast cells were in lung squamous cell carcinoma. There were no significant differences of intratumoural mast cell counts between genders or distinct tumour size groups in adenocarcinoma or in squamous cell carcinoma. In adenocarcinoma, mast cell counts in the well- $(105.1 \pm 71.0)$ and moderate-differentiation subgroups $(77.5 \pm 72.6)$ were significantly higher than in the poor-differentiation subgroup ( $23.0 \pm 19.7 ; \mathrm{p}<0.0005$ and $\mathrm{p}<0.05$, respectively), while there was no significant difference of mast cell counts between the moderate- and well-differentiation subgroups. There was no significant difference of mast cell counts between well- and moderate-differentiation subgroups of lung squamous cell carcinoma. The mast cell count in a case of poorly-differentiated squamous cell carcinoma was one.

\section{Vascular endothelial growth factor expression}

The immunoreactivities of VEGF were found in the tumour cells (fig. 1E and 1F). A part of fibroblasts, smooth muscle cells, and inflammatory cells within the intratumoural stroma were positive for VEGF as well. No significant immunoreactivity was observed when control rabbit IgG was used (fig. $1 \mathrm{G}$ and $1 \mathrm{H}$ ). Immunohistochemical expression of VEGF was found in 33 of 53 cases of stage I lung adenocarcinoma and in 14 of 32 cases of stage I squamous cell carcinoma. There were no significant differences of VEGF expression between genders, distinct tumour size, or distinct histological differentiation subgroups in lung adenocarcinoma or in squamous cell carcinoma.

Double staining of vascular endothelial growth factor and tryptase

This study clearly demonstrates increased accumulation of mast cells within intratumoural stroma. To assess whether intratumoural mast cells express VEGF, the authors double labelled sections with anti-VEGF and with antitryptase antibody (fig. 2A and 2B). It was found that most intratumoural mast cells were positive for VEGF (78$94 \%$ in adenocarcinoma and $75-88 \%$ in squamous cell carcinoma).

Correlations of mast cell counts with microvessel counts

Correlations between mast cell counts and MVCs were analysed at the sites where mast cells most intensively accumulated in NSCLC. Their significant positive correlations were found in lung adenocarcinoma $(\mathrm{r}=0.791 \mathrm{p}<$ $0.0001)$ and squamous cell carcinoma $(r=0.499, p<0.01$; fig. 3). There were significant positive correlations between MVCs at the most neovascularized sites and mast cell counts in lung adenocarcinoma $(\mathrm{r}=0.641, \mathrm{p}<0.0001)$ and squamous cell carcinoma $(\mathrm{r}=0.359, \mathrm{p}<0.05)$. Good correlations were also found between MVCs at the most neovascularized sites and at the sites where mast cells most intensively accumulated in lung adenocarcinoma $(\mathrm{r}=0.859, \mathrm{p}<0.0001)$ and squamous cell carcinoma $(\mathrm{r}=$ $0.637, \mathrm{p}<0.0001)$.

Relationships of vascular endothelial growth factor expression with microvessel counts and mast cell counts

The mean MVC at the most neovascularized sites in VEGF-positive adenocarcinomas $(94.3 \pm 40.6)$ was significantly higher than that in VEGF-negative adenocarcinomas $(56.0 \pm 22.6 ; \mathrm{p}<0.0005)$, whereas there was no significant difference between VEGF-positive (36.7 \pm 16.8$)$ and VEGF-negative squamous cell carcinomas (31.6 \pm 14.9$)$. The mean mast cell count in VEGF-positive adenocarcinomas (102.8 \pm 72.5$)$ was significantly higher than that in VEGF-negative adenocarcinomas $(55.3 \pm 60.6 ; \mathrm{p}<0.005$; fig. 4) whereas there was no significant difference of mast cell counts between VEGF-positive $(16.6 \pm 10.5)$ and VEGF-negative squamous cell carcinomas $(20.3 \pm 18.8)$.

\section{Relationship of clinical outcome to mast cell counts}

By using cut-off values of means of mast cell counts (adenocarcinoma 84.9; squamous cell carcinoma 18.6), the patients were divided into low (adenocarcinoma 30; squamous cell carcinoma 17) and high mast cell count groups (adenocarcinoma 23; squamous cell carcinoma 15).

In patients with lung adenocarcinoma, the postoperative relapse rate in the high mast cell count group $(11 / 23 ; 48 \%)$ 

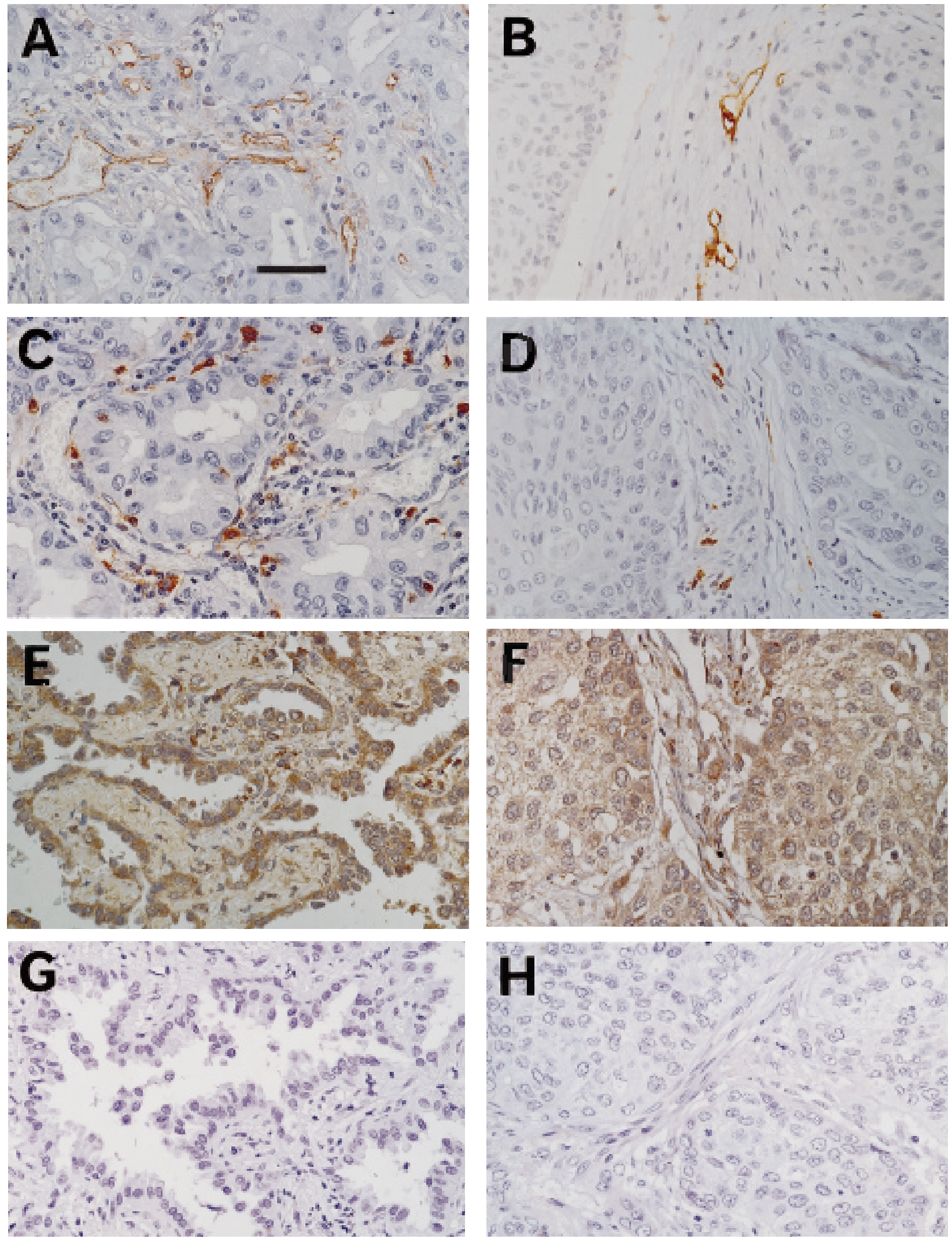

Fig. 1. - Immunohistochemical study of CD34, tryptase and vascular endothelial growth factor (VEGF) in lung adenocarcinoma (A, C, E and G) and squamous cell carcinoma (B, D, F, and H). The delineated CD34-positive cells were assessed as a microvessel (A and B). Tryptase-positive cells are assessed as mast cells (C and D). VEGF was expressed in cancer cells and some stromal cells are positive for VEGF (E and F). No significant immunoreactivity was observed when control rabbit immunoglobulin $\mathrm{G}$ was used $(\mathrm{G}$ and $\mathrm{H})$. Internal scale bar $=50 \mu \mathrm{m}$. 

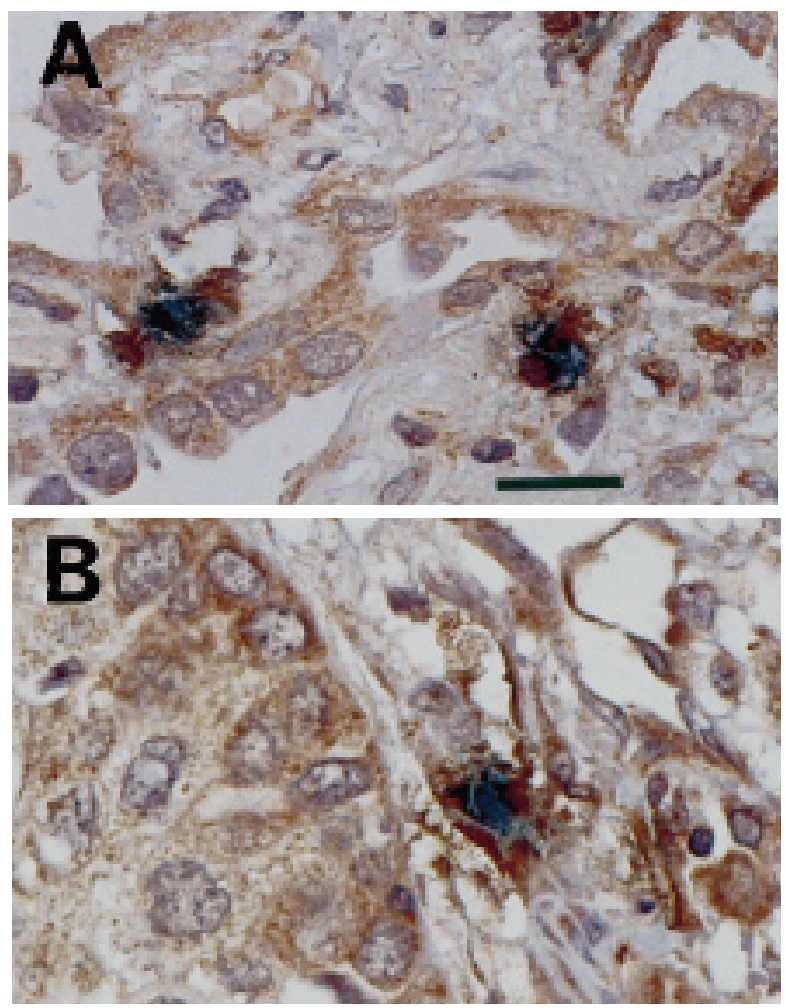

Fig. 2. - Double staining of tryptase and vascular endothelial growth factor (VEGF) in lung adenocarcinoma (A) and squamous cell carcinoma (B). VEGF was visualized using diaminobenzidine as brown and tryptase was visualized using 5-bromo-4-choloro-indolyl phosphate (BCIT)/nitroblue tetrazolium (NBT) as blue in double staining. Tryptase-positive mast cells express VEGF. Internal scale bar $=20 \mu \mathrm{m}$.

was significantly higher than that in the low mast cell count group $(4 / 30 ; 13 \% ; \mathrm{p}<0.01)$ whereas in patients with squamous cell carcinoma there was no significant difference between the low $(5 / 15 ; 33 \%)$ and high mast cell count groups $(6 / 17 ; 35 \%)$. In well- and moderate-differentiation subgroups of lung adenocarcinoma, the postoperative relapse rate in the high mast cell count group $(11 / 23 ; 48 \%)$

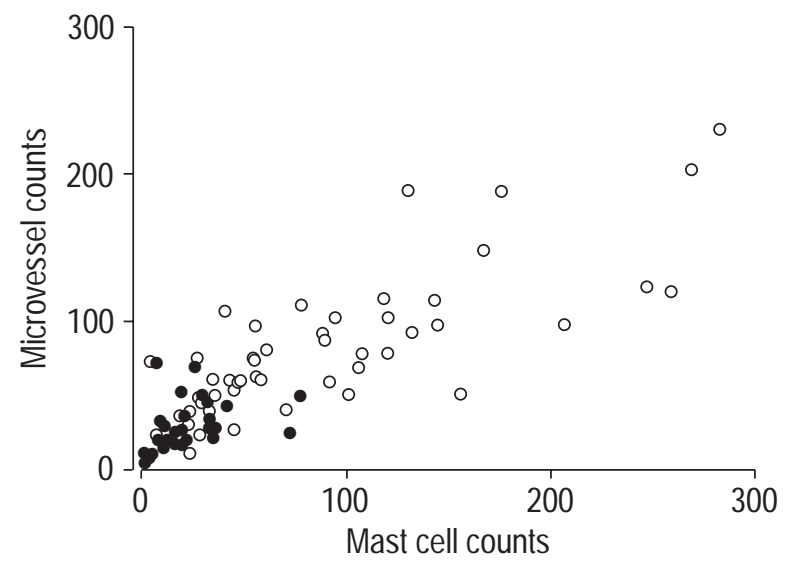

Fig. 3. - Correlations between mast cell counts and microvessel counts (both shown as per $\times 200$ microscopic field) in cases of lung adenocarcinoma $(\bigcirc)$ and squamous cell carcinoma $(\mathbf{O})$. There were significant positive correlations in lung adenocarcinoma $(r=0.791, p<0.0001)$ and squamous cell carcinoma $(\mathrm{r}=0.499, \mathrm{p}<0.01)$.
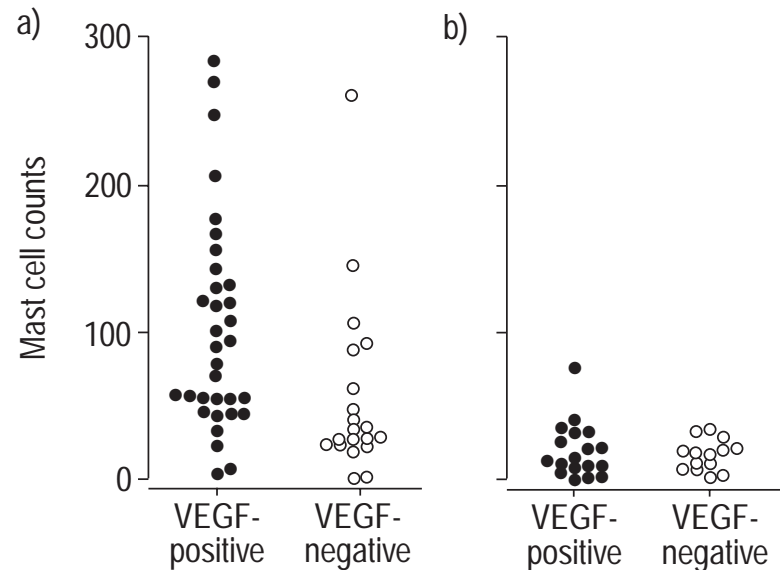

Fig. 4. - Mast cell counts (per $\times 200$ microscopic field) in cases of vascular endothelial growth factor (VEGF)-positive and VEGF-negative lung adenocarcinoma (a) and squamous cell carcinoma (b). In adenocarcinoma mast cell counts in cases of VEGF-positive tumours were significantly higher than those in cases of VEGF-negative tumours $(p<0.005)$, whereas in squamous cell carcinoma they were not.

was significantly higher than that in the low mast cell count group $(2 / 22 ; 9 \% ; \mathrm{p}<0.005)$.

Using the Kaplan-Meier survival estimates, the authors compared survivals in the low and high mast cell count groups. In patients with stage I lung adenocarcinoma. members in the high mast cell count group had significantly worse prognosis than those in the low mast cell count group $(\mathrm{p}<0.05$; fig. $5 \mathrm{a})$, whereas in patients with stage I squamous cell carcinoma, they did not (fig. 5b). In the well- and moderate-differentiation subgroups of lung adenocarcinoma, members in the high mast cell count group had extremely significantly worse prognosis than those in the low mast cell count group ( $<<0.01$; fig. $5 \mathrm{c})$.

\section{Discussion}

This study demonstrates, that counts of intratumoural stromal mast cells significantly correlate with MVCs at the sites where mast cells intensively accumulate in NSCLC. To the authors' knowledge, this is the first report to demonstrate that intratumoural stromal mast cells correlate with angiogenesis in NSCLC. A site where mast cells intensively accumulate may be a site of new microvessel growth in NSCLC and mast cells may play a crucial role in neovascularization in NSCLC, especially lung adenocarcinoma. Angiogenesis has much to do with mast cells, because mast-cell-deficient W/Wv mice growing B16-BL6 tumours have been shown to exhibit a decreased rate of tumour angiogenesis as compared with mast-cell-sufficient +/+ littermate mice [19].

Neovascularization is crucial for sustained tumour growth as it allows oxygenation and nutrient perfusion of the tumour as well as removal of waste products. Increased angiogenesis coincides with increased tumour cell entry into circulation and thus facilitates metastasis $[1,2]$ Angiogenesis assessed by MVCs is related with poor outcome in stage I NSCLC [4-7]. To date, at least 20 angiogenic molecules have been identified. Among these molecules with angiogenic activity, VEGF is thought to be one of the most important, because specific inhibition of VEGF decreases tumour neovascularization and 

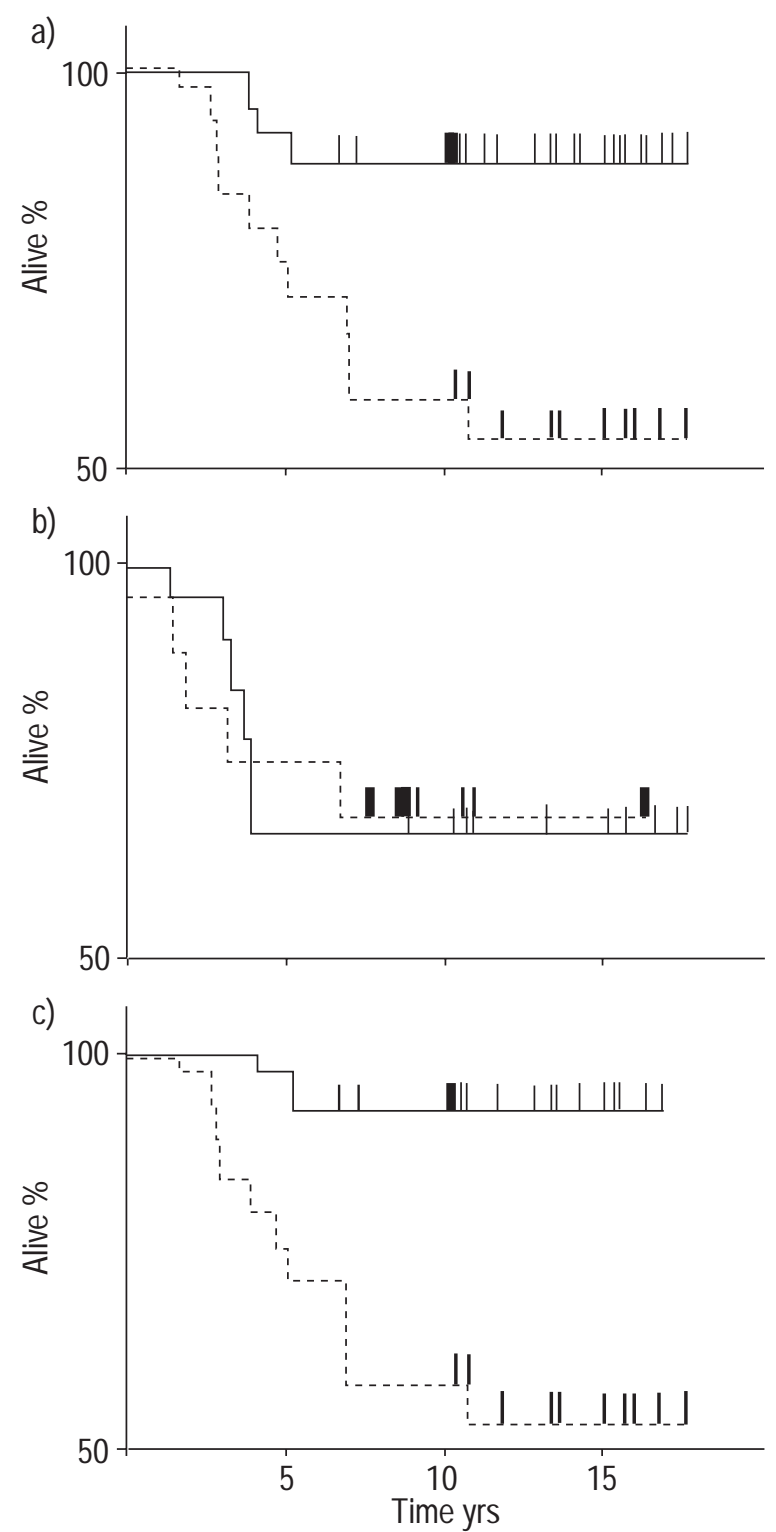

Fig. 5. - Kaplan-Meier survival estimates stratified by mast cell counts in patients with lung adenocarcinoma ( $\mathrm{a}$ and $\mathrm{c}$ ) and squamous cell carcinoma (b). a and b show all patients and c shows well- and moderatedifferentiation subgroups of lung adenocarcinoma. The Wilcoxon test showed a statistically significant difference between two groups in patients with stage I lung adenocarcinoma $(\mathrm{p}<0.05)$ and with stage I well- and moderately-differentiated lung adenocarcinoma $(\mathrm{p}<0.01)$ and no statistically significant difference between two groups in patients with stage I lung squamous cell carcinoma. a) - : mast cell count $<84.9, \mathrm{n}=30 ;-\cdots$ - : mast cell count: $\geq 84.9, \mathrm{n}=23$. b) - : $_{\text {mast cell }}$

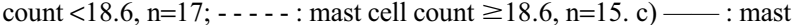
cell count $<84.9, \mathrm{n}=22$; - - - - : mast cell count $\geq 84.9, \mathrm{n}=23$.

substantially inhibits primary tumour growth in vivo [23]. The current authors and other investigators have detected VEGF expression in NSCLC $[4,6,7]$.

YUAN and coworkers [3] and SHIJUBo et al. [7] have demonstrated that the mean MVC in lung adenocarcinoma is significantly higher than in lung squamous cell carcinoma. This study demonstrates significantly higher counts of intratumoural stromal mast cells in lung adenocarcinoma than those in lung squamous cell carcinoma. In lung adenocarcinoma, mast cell counts in the well- and moderate-differentiation subgroups were significantly higher than in the poor-differentiation subgroup. The difference of mast cell counts between lung adenocarcinoma and squamous cell carcinoma and among histological differentiation subgroups of lung adenocarcinoma may be elucidated by the complex molecular and cellular mechanisms of mast cell accumulation within tumours. The difference of mast cell accumulation between lung adenocarcinoma and squamous cell carcinoma may be elucidated by the differing production of the cytokines that have properties that reduce mast cell accumulation. Slow tumour growth seems to make a good environment for slow activation of mast cells and accumulation of mast cells. Interestingly, the number of mast cells in the upper dermis was significantly increased in VEGF transgenic mice using a keratin 14 promoter expression cassette containing the gene for murine VEGF-164 to selectively target VEGF expression to basal epidermal keratinocytes [24], suggesting that VEGF overproduction from keratinocytes induces mast cell migration. A recent study [21] has demonstrated that mast cells migrate in response to angiogenic factors including VEGF. In lung adenocarcinoma, VEGF-positive tumours had significantly higher mast cell counts than VEGF-negative tumours, whereas in lung squamous cell carcinoma they did not. The evidence suggests that unlike in lung squamous cell carcinoma, VEGF produced from lung adenocarcinoma cells may induce mast cell migration, although the mechanisms by which mast cells accumulate within tumours remain unclear. Further investigations will be needed to elucidate why VEGF induces angiogenesis and mast cell accumulation in lung adenocarcinoma but not in lung squamous cell carcinoma.

Mast cells accumulate in a number of angiogenesisdependent situations including rheumatoid arthritis [25], wound healing [26], and malignant tumours [8-13]. In vitro, mast cell conditioned medium stimulates capillary endothelial cell migration [14]. Mast cell granules localize within endothelial cells and stimulate their proliferation [14]. Mast cell products also degrade connective tissue matrix to provide space for neovascular sprouts to form [16]. Mast cell histamine induces angiogenesis and heparin promotes new vessel formation in vivo [14]. Tryptase derived from mast cells stimulates vascular tube formation and functions as a mitogen for microvascular endothelial cells [16]. Tryptase also activates latent metalloproteinases [27] and plasminogen activator [14], which function to degrade the extracellular matrix. Mast cells have been reported to express matrix metalloproteases 2 and 9 [28], which are pivotal enzymes in matrix remodelling. Such matrix degradation is crucial in the early stages of angiogenesis. Mast cells have been shown to produce VEGF $[17,18]$. This article documents VEGF expression in intratumoural stromal mast cells in stage I NSCLC.

Increased numbers of mast cells have been shown in colorectal cancer [8], breast carcinoma $[9,10]$, basal cell carcinoma of the skin [11], soft tissue tumours [12] and melanoma [13]. The functional significance of the accumulation of mast cells within tumours is a subject of controversy because of contradictory experimental [29] and clinical data; intratumoural mast cell numbers correlate with metastasis in breast carcinoma [9] and invasiveness in melanoma [13], while in contrast in soft tissue sarcoma, patients with distant metastases all belonged to 
the low mast cell count group [12]. In mast-cell-deficient $\mathrm{W} / \mathrm{Wv}$ mice a decreased rate of tumour angiogenesis has been shown in parallel with a decreased haematogenous metastasis [19]. However, mast cells are shown to be a rich source of tumour necrosis factor- $\alpha$ cytotoxic for some tumours [14, 29], and the mast cell derived cytokine, interleukin-4, which inhibits growth and induces apoptosis in human carcinoma cells [30]. In patients with stage I lung adenocarcinoma, the postoperative relapse rate in the high mast cell count group was significantly higher than in the low mast cell count group, whereas there was no significant difference of the postoperative relapse rate between two groups in stage I lung squamous cell carcinoma. More importantly, only in patients with stage I lung adenocarcinoma, the high mast cell count group had significantly worse prognosis than the low mast cell count group. This is the first report to document that mast cell accumulation within tumours correlates with metastasis and poor outcome in patients with lung adenocarcinoma.

It is concluded that intratumoural stromal mast cells correlate with angiogenesis assessed by microvessel counts and with poor outcome in stage I lung adenocarcinoma. Vascular endothelial growth factor released from adenocarcinoma cells may be related to mast cell accumulation and most intratumoural stromal mast cells produce vascular endothelial growth factor. Intratumoural stromal mast cell counts may be a significant prognostic factor in patients with stage I well- and moderately-differentiated lung adenocarcinoma.

\section{References}

1. Follman J, Whatson K, Ingber D, Hanahan D. Induction of angiogenesis during the transition from hyperplasia to neoplasia. Nature 1989; 339: 58-61.

2. Weidner N, Semple JP, Welch WR, Follman J. Tumor angiogenesis and metastasis-correlation in invasive breast carcinoma. N Engl J Med 1991; 324: 1-8.

3. Yuan A, Yang PC, Yu CJ, et al. Tumor angiogenesis correlates with histologic type and metastasis in nonsmallcell lung cancer. Am J Respir Crit Care Med 1995; 152: 2157-2162.

4. Fontanini G, Vignati S, Lucchi M, et al. Neoangiogenesis and 553 protein in lung cancer: their prognostic role and their relation with vascular endothelial growth factor (VEGF) expression. Br J Cancer 1997; 75: 1295-1301.

5. Duarte IG, Bufkin BL, Pennington MF, et al. Angiogenesis as a predictor of survival after surgical resection for stage I non-small-cell lung cancer. J Thorac Cardiovasc Surg 1998; 115: 652-658.

6. Shibusa T, Shijubo N, Abe S. Tumor angiogenesis and vascular endothelial growth factor expression in stage I lung adenocarcinoma. Clin Cancer Res 1998; 4: 14831487.

7. Shijubo N, Uede T, Kon S, et al. Vascular endothelial growth factor and osteopontin in stage I lung adeno-carcinoma. Am J Respir Crit Care Med 1999; 160: 1269 1273 .

8. Lachter J, Stein M, Lichtig C, Eidelman S, Munichor M. Mast cells in colorectal neoplasias and premalignant disorders. Dis Colon Rectum 1995; 38: 290-293.

9. Hartveit F, Thoresen S, Tangen M, Maartmann-Moe H. Mast cell changes and tumour dissemination in human breast carcinoma. Invasion Metastasis 1984; 4: 146-155.

10. Kankkunen JP, Harvima IT, Naukkarinen A. Quantitative analysis of tryptase and chymase containing mast cells in benign and malignant breast lesions. Int J Cancer 1997; 72: $385-388$.
11. Yamamoto T, Katayama I, Nishioka K, Duncan LM, Richards LA, Mihm MC Jr. Expression of stem cell factor in basal cell carcinoma. Br J Dermatol 1997; 137: 709713.

12. Donhuijsen K, Sastry M, Volker B, Leder LD. Mast cell frequency in soft tissue tumors: relation to type and grade of malignancy. Pathol Res Prac 1992; 188: 61-66.

13. Duncan LM, Richards LA, Mihm MC Jr. Increased mast cell density in invasive melanoma. J Cutan Pathol 1998; 25: $11-15$.

14. Metcalfe DD, Baram D, Mekori YA. Mast cells. Physiol Rev 1997; 77: 1033-1079.

15. Meininger CJ, Zetter BR. Mast cells and angiogenesis. Semin Cancer Biol 1992; 3: 73-79.

16. Blair RJ, Meng H, Marchese MJ, et al. Human mast cells stimulate vascular tube formation: tryptase is a novel, potent angiogenic factor. J Clin Invest 1997; 99: 26912700.

17. Grutzkau A, Kruger-Krasagakes S, Baumeister H, et al. Synthesis, storage, and release of vascular endothelial growth factor/ vascular permeability factor (VEGF/VPF) by human mast cells: implications for the biological significance of VEGF206. Mol Biol Cell 1998; 9: 875884.

18. Boesiger J, Tsai M, Maurer $\mathrm{M}$, et al. Mast cells can secrete vascular permeability factor/vascular endothelial cell growth factor and exhibit enhanced release after immunoglobulin E-dependent upregulation of $\mathrm{fc}$ epsiion receptor 1 exoression. J Exp Med 1998; 188: 1135-1145.

19. Starkey JR, Crowle PK, Taubenberger S. Mast-celldeficient $\mathrm{W} / \mathrm{Wv}$ mice exhibit a decreased rate of tumor angiogenesis. Int J Cancer 1988; 42: 8-52.

20. Neufeld G, Cohen T, Gengrinovitch S, Poltorak Z. Vascular endothelial growth factor (VEGF) and its receptors. FASEB J 1999; 13: 9-22.

21. Gruber BL, Marchese MJ, Kew R. Angiogenic factors stimulate mast-cell migration. Blood 1995; 86: 2488-2493.

22. Shijubo N, Itoh Y, Yamaguchi T, et al. Clara cell proteinpositive epithelial cells are reduced in small airways of asthmatics. Am J Respir Crit Care Med 1999; 160: 930933.

23. Kondo S, Asano M, Suzuki H. Significance of vascular endothelial growth factor/vascular permeability factor for solid tumor growth, and its inhibition by the antibody. Biochem Biophys Res Commun 1993; 94: 1234-1241.

24. Detmar M, Brown LF, Schon MP, et al. Increased microvascular density and enhanced leukocyte rolling and adhesion in the skin of VEGF transgenic mice. Invest Dermatol 1998; 111: 1-6.

25. Malone DG, Irani AM, Schwartz LB, Barrett KE, Metcalfe DD. Mast cell numbers and histamine levels in synovial fluids from patients with diverse arthritides. Arthritis Rheum 1986; 29: 956-963.

26. Trabucchi E, Radaelli E, Marazzi M, et al. The role of mast cells in wound healing. Int J Tissue React 1988; 10 : 367-372.

27. Gruber BL, Marchese MJ, Suzuki K, et al. Synovial procollagenase activation by human mast cell tryptase dependence upon matrix metalloproteinase 3 activation. $J$ Clin Invest 1989; 84: 1657-1662.

28. Fang KC, Wolters PJ, Steinhoff M, Bidgol A, Blount JL, Caughey GH. Mast cell expression of gelatinases A and B is regulated by kit ligand and TGF- $\beta$. J Immunol 1999; 162: 5528-5535.

29. Dimitiradou V, Koutsilieris M. Mast cell-tumor interactions: for or against tumour growth and metastasis. $A n-$ ticancer Res 1997; 17: 1541-1549.

30. Gooch JL, Lee AV, Yee D. Interleukin 4 inhibits growth and induces apoptosis in human breast cancer cells. Cancer Res 1998; 58: 4199-4205. 